Keywords Cournot duopoly game; best response mechanism; bounded rationality; heterogeneous players; bifurcation; complex dynamics

Abstract In this paper we propose and compare three heterogeneous Cournotian duopolies, in which players adopt best response mechanisms based on different degrees of rationality. The economic setting we assume is described by an isoelastic demand function with constant marginal costs. In particular, we study the effect of the rationality degree on stability and convergence speed to the equilibrium output. We study conditions required to converge to the Nash equilibrium and the possible route to destabilization when such conditions are violated, showing that a more elevated degree of rationality of a single player does not always guarantee an improved stability. We show that the considered duopolies exhibit either a flip or a Neimark-Sacker bifurcation. In particular, in heterogeneous oligopolies models, the Neimark-Sacker bifurcation usually arises in the presence of a player adopting gradient-like decisional mechanisms, and not best response heuristic, as shown in the present case. Moreover, we show that the cost ratio crucially influences not only the size of the stability region, but also the speed of convergence toward the equilibrium. 


\title{
Nonlinear dynamics and convergence speed of heterogeneous Cournot duopolies involving best-response mechanisms with different degrees of rationality
}

\author{
Fausto Cavalli · Ahmad Naimzada
}

the date of receipt and acceptance should be inserted later

\section{Introduction}

A duopoly is a market supplied by only two firms. Cournot [12] developed the first formal theory of duopolistic markets and introduced a model which is still an interesting subject of economic dynamics and game theory study. An oligopolistic market is much more complex that a monopolistic or competitive one, as the firms, to make choices, in addition to the demand function, have to consider at the same time both their own and their opponents decisions. Such framework involves a wide range of computational and informational capabilities, and it can be not realistic to assume full rationality for all the firms, which would give rise to a static model.

Rand [26] and Poston and Stewart [23] considered a simple duopoly, with abstractly set reaction functions, which could give rise to complex dynamic phenomena. This strand was continued by Puu [24], in which the reaction functions were rigorously derived by solving an optimization problem for the profit functions. Puu considered the case of constant marginal costs and isoelastic demand function, microfunded on Cobb-Douglas consumer preferences. He showed that the outputs evolution of each competitor can lead to a period doubling sequence of flip bifurcations and then to chaos. Recently, the best response decisional mechanism has also been considered in a setting with sequential decisions by Gao et al. [16].

Progressively, the research aims focused on investigating different decisional mechanisms, involving low degrees of rationality of the players. We can mention the gradient-like mechanism proposed by Bischi and Naimzada [8], Bischi et al. [6, 7] and Agiza et al. [3], Askar [5], in which no optimization problem is solved by players, which only adjust their strategies using a local estimate of the marginal profit. Another decisional mechanism (introduced by Silvestre [27] and reconsidered by Tuinstra [29], Bischi et al. [9], Naimzada and Sbragia [21], Naimzada and Ricchiuti [20]) is the "Local Monopolistic Approximation" (LMA), in which

F. Cavalli A. Naimzada

Department of Economics, Management and Statistics, University of Milano-Bicocca, Piazza dell'Ateneo Nuovo, 1, 20126 Milan, Italy

E-mail: fausto.cavalli@unimib.it

E-mail: ahmad.naimzada@unimib.it 
oligopolists conjecture a linear approximation of the demand function, by means of a local knowledge of true demand curve and the market state in terms of quantities and price. Finally, several authors studied what happens when heterogeneous firms, adopting different decisional mechanisms, are coupled. We mention the works by Leonard and Nishimura [17], Den-Haan [13], Agiza and Elsadany [1,2], Agnelini et al. [4], Tramontana [28], Dubiel-Teleszynski [14], Cavalli et al. [10,11]. Finally, the study of heterogeneity in oligopolies with more than two firms is conducted for example in the contributions by $\mathrm{Li}$ and $\mathrm{Ma}$ [18], $\mathrm{Ma}$ and $\mathrm{Wu}$ [19], Elsadany and Tramontana [15].

The main purpose of this paper is to compare several dynamic duopolistic Cournotian games, which differentiate themselves for the degree of rationality of the players. In particular, we consider rational players, which are able to compute the best response function and have perfect foresight, in the sense that their expectation about the strategy of the other player for the next period is correct, naive players, which are able to compute correctly the best response function but they have not perfect foresight and so they adopt static expectations, and reduced rationality or LMA players, which use the "Local Monopolistic Approximation".

We set in the research strand of heterogeneous oligopolies, and we combine in different ways the previous players, considering three distinct duopolies. In the first one we couple a rational player with a naïve one, in the second one we couple a rational player with a reduced rationality one, in the third one we couple a naïve player with a reduced rationality one, so that the duopolies actually differ for the degree of rationality of the involved players. The economic setting is defined by an isoelastic demand function and we assume linear total costs for the firms.

The main results concern the study of the effect of the rationality on the stability and the convergence speed to the equilibrium output. Regarding the stability, we investigate both the conditions required to converge to the Nash equilibrium and the possible route to destabilization when such conditions are violated. In particular, we show that the presence of the LMA mechanism has a stabilizing effect. Equally, in the studied examples, an elevated degree of rationality does not necessarily implies a reduced region of stability. Conversely, stability is governed by the marginal cost ratio, which, if sufficiently unbalanced, introduces instability. We show that all the considered duopolies exhibit a flip bifurcation except for the duopoly with naïve and LMA players, in which the equilibrium loses stability through a Neimark-Sacker. This is a significantly different behavior from the Puu model in which, although sharing the economic setting, stability is lost through a flip bifurcation. This behavior is particularly interesting also compared to the existing literature, as the Neimark-Sacker bifurcation is usually seen in the presence of firms adopting a gradient-like decisional process $[6,7,4,28,10]$. On the contrary, in the present work, we show that such destabilization can occur with mechanisms based on the best response too.

Moreover, we prove that the cost ratio influences in a determinant way not only the size of the stability region, but also the speed of convergence toward the equilibrium, namely the number of iterations required to approach the equilibrium with a certain accuracy.

The paper is organized as follows. In Section 2 we introduce the duopolistic Cournotian game. In Section 3 we present the different decisional mechanisms and the models we want to study. In Section 4 we study the nonlinear stability and in 
Section 5 we investigate the speed of convergence. Conclusions and perspectives are presented in Section 6 .

\section{Duopolistic Cournotian game}

We consider an economy populated by $n$ agents. Let $q_{j}=\left(q_{j}^{1}, q_{j}^{2}, \ldots, q_{j}^{m}\right)$ be the vector of the quantities of $m$ goods. Assuming Cobb-Douglas preferences for the agents, with utility functions $U_{j}\left(q_{j}\right)=\prod_{k=1}^{m}\left(q_{j}^{k}\right)^{\alpha_{j}^{k}}$, if we indicate with $p_{k}$ the price of commodity $k$ and with $y^{j}$ the income of the $j$ th agent, under the budget constraint $\sum_{k=1}^{m} p_{k} q_{j}^{k} \leq y^{j}$, we obtain

$$
q_{j}^{k}=\frac{\alpha_{j}^{k} y^{j}}{p_{k}}
$$

by solving a constrained maximization problem. Let us focus on just one market, so that we can suppress the index $k=1, \ldots, m$. Introducing the aggregated demand $Q=\sum_{j} q_{j}$, we obtain the aggregated inverse demand function

$$
p(Q)=\frac{1}{Q}
$$

where we set $\sum_{j} \alpha_{j} y^{j}=1$. We remark that the resulting inverse demand function (1) is the same isoelastic one studied by Puu [24].

In the present work, the industry producing the good we are considering is made up by two firms (indexed by $i=1,2$ ), which produce and supply two homogeneous goods $q_{1}$ and $q_{2}$ and have linear cost functions

$$
C_{i}\left(q_{i}\right)=c_{i} q_{i}, i=1,2,
$$

where $c_{i}>0$ represent the (constant) marginal cost of each duopolist. The resulting profit functions of the firms are then

$$
\Pi_{i}\left(q_{i}, Q\right)=\frac{q_{i}}{Q}-c_{i} q_{i}, i=1,2
$$

We then have a game, in which players are the two duopolists, the feasible strategies are the nonnegative production levels $\left(q_{i} \geq 0\right)$ and the payoff functions are the profit functions (2). In this work, to focus on a true duopolistic market, we only consider strictly positive output levels, namely $q_{i}>0$, which means that both the firms are producing.

As in the work by Puu [24], we have a unique positive Nash equilibrium given by

$$
q^{N}=\left(q_{1}^{N}, q_{2}^{N}\right)=\left(\frac{c_{2}}{\left(c_{1}+c_{2}\right)^{2}}, \frac{c_{1}}{\left(c_{1}+c_{2}\right)^{2}}\right)
$$

We underline that, when equilibrium (3) is achieved, the output produced by the most efficient firm is larger than the opponent's one, as well as its profits. 


\section{Dynamic Models}

The Nash equilibrium notion is very demanding in terms of rationality and information set, since players have to exactly know the demand function and to correctly forecast the competitor decisions. If all the players were endowed with such degree of rationality, the Nash equilibrium would be achieved in just one-shot. So it is natural to assume that agents have reduced informational and computational capabilities. This implies that, in order to make choices, agents have to consider past market outcomes, i.e. past strategies, prices and profits. This gives rise to a dynamical adjustment process.

We start describing three players which, although using decisional mechanisms based on the best response method, are endowed with different degrees of rationality. The first two players we consider are rational and naïve players, who both have sufficient informational and computational capabilities to optimally respond to the other player strategy. In particular, both players know the demand function, the cost function and they are able to compute the output level $q_{i, t+1}$, which, on the base of the expected strategy of the other player, maximize their profits. Since the marginal profit of the $i$ th agent is

$$
\phi_{i}\left(q_{i}, Q\right)=\partial_{q_{i}} \Pi_{i}\left(q_{i}, Q\right)=\frac{Q-q_{i}}{Q^{2}}-c_{i}
$$

the optimization problem is obtained by imposing

$$
\phi_{i}\left(q_{i, t+1}, Q_{t+1}^{e}\right)=0,
$$

where $Q_{t+1}^{e}=q_{i, t+1}+q_{-i, t+1}^{e}$ is the expected aggregated strategy, which depends on the strategy $q_{-i, t+1}^{e}$ that the $i$ th firm expects that its opponent $-i$ will choose at time $t+1$. By obtaining $q_{i, t+1}$ from (4), we have the best response function

$$
q_{i, t+1}=\sqrt{\frac{q_{-i, t+1}^{e}}{c_{i}}}-q_{-i, t+1}^{e} .
$$

Rational and naïve players differ with respect to the expected strategy. Rational player has perfect foresight and he is able to correctly foresee the next period strategy of his opponent, so that $q_{-i, t+1}^{e}=q_{-i, t+1}$. In this case equation (5) becomes

$$
q_{i, t+1}=\sqrt{\frac{q_{i, t+1}}{c_{i}}}-q_{i, t+1}
$$

Conversely, naïve player assumes static expectations $q_{-i, t+1}^{e}=q_{-i, t}$ and consequently (5) becomes

$$
q_{i, t+1}=\sqrt{\frac{q_{-i, t}}{c_{i}}}-q_{-i, t} .
$$

The last economic agent we consider has further reduced rationality. In particular, he has only a local knowledge of the demand function and adopts the so-called "Local Monopolistic Approximation" (LMA). The LMA [21,9,22] is a bounded rational adjustment process, in which the firm has only a limited knowledge of the demand function, in particular it knows the market price $p_{t}$, the corresponding produced quantity $Q_{t}$, and is able to obtain a correct estimate of the slope of the price function in $\left(p_{t}, Q_{t}\right)$. As noticed in [9], such estimation can be obtained 
through market research, looking for a correct local estimate of the price function for market values in a neighborhood of $\left(p_{t}, Q_{t}\right)$ in order to correctly compute the derivative $f^{\prime}\left(Q_{t}\right)$. Then, the firm uses such information to conjecture the demand function by means of a linear approximation of the true demand function in $\left(p_{t}, Q_{t}\right)$ obtaining the expected price function

$$
p_{i, t+1}^{e}=p_{i}\left(Q_{t}\right)+p^{\prime}\left(Q_{t}\right)\left(Q_{t+1}^{e}-Q_{t}\right) .
$$

Assuming static expectations $\left(q_{-i, t+1}^{e}=q_{-i, t}\right)$, we can rewrite (8) as

$$
p_{i, t+1}^{e}=\frac{1}{Q_{t}}-\frac{1}{\left(Q_{t}\right)^{2}}\left(q_{i, t+1}-q_{i, t}\right),
$$

where we also used (1). By means of the globally conjectured demand function the firm optimizes the expected profits: in order to compute the best response on the base of the conjectured price (9), the player has to solve

$$
q_{i, t+1}=\arg \max _{q_{i, t+1}}\left[p_{i, t+1}^{e} q_{i, t+1}-c_{i} q_{i, t+1}\right]
$$

which gives

$$
q_{i, t+1}=\frac{1}{2} q_{i, t}+\frac{1}{2}\left(1-c_{i}\left(q_{i, t}+q_{-i, t}\right)\right)\left(q_{i, t}+q_{-i, t}\right) .
$$

We notice that, for some $q_{i, t}$, both (6), (7) and (10) could give negative output levels, which means that the output level should actually be null. However, as already remarked, in this work we only focus on positive output levels, so we restrict ourselves to initial data that produce positive trajectories.

\subsection{Heterogeneous duopolies}

The oligopolistic competitions we want to focus on are those heterogeneous, in the sense that the players do not adopt the same heuristic.

The duopoly involving the most elevated degree of rationality is obtained by coupling a rational and a naïve player, and it is modeled by

$$
T_{R B}\left(q_{1}, q_{2}\right):\left\{\begin{aligned}
q_{1, t+1} & =\sqrt{\frac{q_{2, t}}{c_{1}}}-q_{2, t}, \\
q_{2, t+1} & =\sqrt{\frac{q_{1, t+1}}{c_{2}}}-q_{1, t+1} .
\end{aligned}\right.
$$

which actually reduces to the one dimensional map

$$
T_{R B}\left(q_{1}\right): q_{1, t+1}=\sqrt{\frac{1}{c_{1}}\left(\sqrt{\frac{q_{1, t}}{c_{2}}}-q_{1, t}\right)}-\sqrt{\frac{q_{1, t}}{c_{2}}}+q_{1, t},
$$

Hereinafter, we will indicate both (11) and (12) by RB.

The second duopoly we consider is obtained coupling a rational player and a LMA player

$$
T_{R L}\left(q_{1}, q_{2}\right):\left\{\begin{array}{l}
q_{1, t+1}=\frac{1}{2} q_{1, t}+\frac{1}{2}\left(1-c_{1}\left(q_{1, t}+q_{2, t}\right)\right)\left(q_{1, t}+q_{2, t}\right), \\
q_{2, t+1}=\sqrt{\frac{q_{1, t+1}}{c_{2}}}-q_{1, t+1},
\end{array}\right.
$$


which can again be studied by means of the one-dimensional map

$$
T_{R L}\left(q_{1}\right): q_{1, t+1}=\frac{1}{2} q_{1, t}\left(1-\frac{c_{1}}{c_{2}}\right)+\frac{1}{2} \sqrt{\frac{q_{1, t}}{c_{2}}} .
$$

We make reference to such model using RL.

In the last duopoly, we couple the two least rational players, naïve and LMA player, obtaining the following two dimensional discrete dynamical system

$$
T_{B L}\left(q_{1}, q_{2}\right):\left\{\begin{array}{l}
q_{1, t+1}=\frac{1}{2} q_{1, t}+\frac{1}{2}\left(1-c_{1}\left(q_{1, t}+q_{2, t}\right)\right)\left(q_{1, t}+q_{2, t}\right), \\
q_{2, t+1}=\sqrt{\frac{q_{1, t}}{c_{2}}}-q_{1, t},
\end{array}\right.
$$

which will be identified with BL.

Finally, as a comparison, we consider the classical (homogeneous) model studied by Puu [24]

$$
T_{B B}\left(q_{1}, q_{2}\right):\left\{\begin{aligned}
q_{1, t+1} & =\sqrt{\frac{q_{2, t}}{c_{1}}}-q_{2, t} \\
q_{2, t+1} & =\sqrt{\frac{q_{1, t}}{c_{2}}}-q_{1, t}
\end{aligned}\right.
$$

We will refer to (15) with $\mathrm{BB}$.

\section{Analysis}

We start considering the possible steady states for RB, RL and BL models. We have the following straightforward result.

Proposition 1 Both (12), (13) and (14) have a unique positive steady state which coincides with the Nash equilibrium (3).

Before studying local stability of RB, BL and RL, we recall [24] that, for the BB model, the Nash equilibrium is locally stable provided that

$$
3-2 \sqrt{2}<\frac{c_{1}}{c_{2}}<3+2 \sqrt{2} .
$$

$R B$ model local stability analysis

Map (12) is unimodal when $c_{1} / c_{2} \leq 1$ and bimodal when $c_{1} / c_{2}>1$, as shown in Figure 1. Local stability is studied in the following

Proposition 2 The Nash equilibrium is locally asymptotically stable provided that

$$
3-2 \sqrt{2}<\frac{c_{1}}{c_{2}}<3+2 \sqrt{2} .
$$

Proof The steady state is stable provided that $\left|T_{R B}^{\prime}\left(q^{N}\right)\right|<1$. From

$$
T_{R B}^{\prime}\left(q_{1}\right)=\left(\frac{1}{2 \sqrt{c_{2} q_{1}}}-1\right)\left(\frac{1}{2 \sqrt{c_{1}\left(\sqrt{\frac{q_{1}}{c_{2}}}-q_{1}\right)}}-1\right),
$$



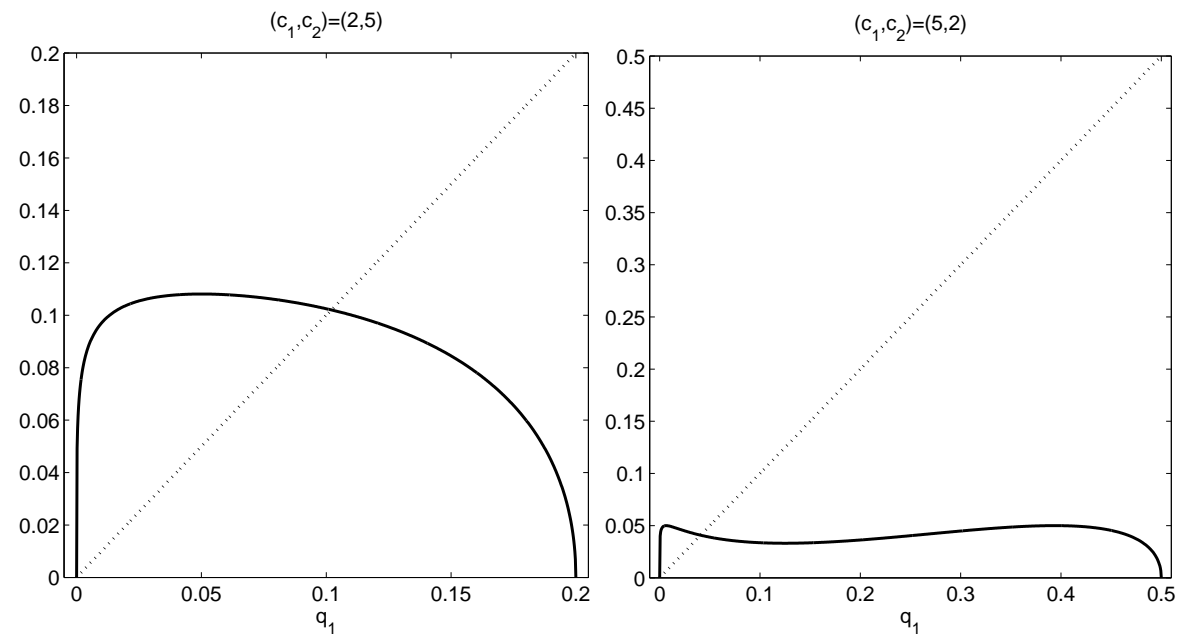

Fig. 1 Plot of RB map. When $c_{1} / c_{2} \leq 1$ (left) the map is unimodal, while when $c_{1} / c_{2}>1$ (right) it is bimodal.

we have

$$
T_{R B}^{\prime}\left(q^{N}\right)=-\frac{\left(c_{1}-c_{2}\right)^{2}}{4 c_{1} c_{2}} .
$$

Since $T_{R B}^{\prime}\left(q^{N}\right)<0$, we only need $T_{R B}^{\prime}\left(q^{N}\right)>-1$, which is equivalent to $c_{1}^{2}-$ $6 c_{1} c_{2}+c_{2}^{2}<0$. This allows concluding.

\section{$R L$ model local stability analysis}

Map (13) is strictly increasing when $c_{1} / c_{2} \leq 1$, while it is unimodal when $c_{1} / c_{2}>1$, as shown in Figure 2. We have the following stability result.

Proposition 3 The Nash equilibrium is locally asymptotically stable for

$$
0<\frac{c_{1}}{c_{2}} \leq 7
$$

Proof Since

$$
T_{R L}^{\prime}\left(q^{N}\right)=\frac{3}{4}-\frac{c_{1}}{4 c_{2}},
$$

we have that $\left|T_{R L}^{\prime}\left(q^{N}\right)\right|<1$ for $c_{1} / c_{2}<7$, which allows concluding.

Thanks to the previous results, we have again that when the iterated map converges, the limit is the Nash equilibrium.

\section{BL model local stability analysis}

To investigate the local stability of the equilibrium, we need the Jacobian matrix of system (14)

$$
J\left(q_{1}, q_{2}\right)=\left(\begin{array}{cc}
1-c_{1}\left(q_{1}+q_{2}\right) & \frac{1}{2}-c_{1}\left(q_{1}+q_{2}\right) \\
\frac{1}{2 \sqrt{q_{1} c_{2}}}-1 & 0
\end{array}\right) .
$$



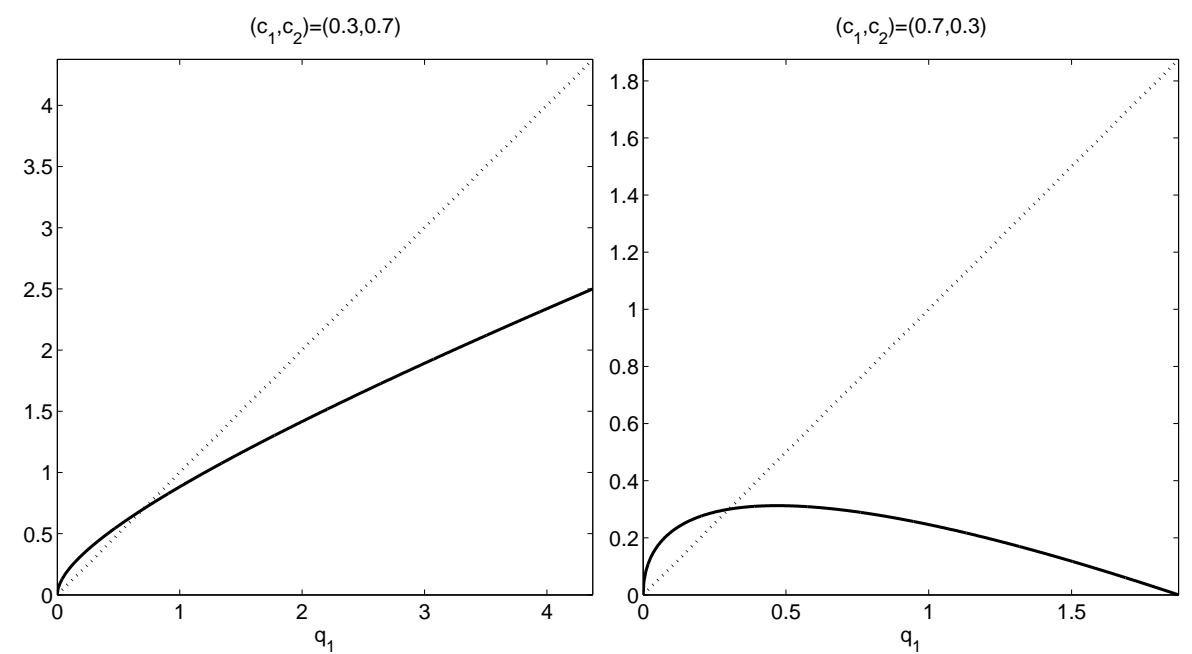

Fig. 2 Plot of RL map. When $c_{1} / c_{2} \leq 1$ (left) the map is increasing, while when $c_{1} / c_{2}>1$ (right) it is unimodal.

Recalling Jury's condition for the local stability of a steady state

$$
\left\{\begin{array}{l}
1-\operatorname{Tr}\left(J\left(q^{N}\right)\right)+\operatorname{det}\left(J\left(q^{N}\right)\right)>0 \\
1+\operatorname{Tr}\left(J\left(q^{N}\right)\right)+\operatorname{det}\left(J\left(q^{N}\right)\right)>0 \\
1-\operatorname{det}\left(J\left(q^{N}\right)\right)>0
\end{array}\right.
$$

we have the following local stability result.

Proposition 4 The Nash equilibrium (3) is locally asymptotically stable provided that

$$
0<\frac{c_{1}}{c_{2}}<3+2 \sqrt{3}
$$

Proof Evaluating the Jacobian matrix in the Nash equilibrium (3), we obtain

$$
J\left(q^{N}\right)=\left(\begin{array}{cc}
\frac{c_{2}}{c_{1}+c_{2}} & \frac{c_{1}-c_{2}}{2\left(c_{1}+c_{2}\right)} \\
\frac{c_{1}-c_{2}}{2 c_{2}} & 0
\end{array}\right),
$$

and, consequently,

$$
\operatorname{Tr}(J)=\frac{c_{2}}{c_{1}+c_{2}}, \quad \operatorname{det} J=\frac{\left(c_{1}-c_{2}\right)^{2}}{4 c_{2}\left(c_{1}+c_{2}\right)} .
$$

The first two conditions of (21) are automatically fulfilled, while the third one requires that $c_{2} / c_{1} \in(3-2 \sqrt{3}, 3+2 \sqrt{3})$, which, since $c_{i}>0$, reduces to $(22)$.

The previous proposition allows concluding that if (14) converges, then the limit is the Nash equilibrium. Moreover, when condition (22) is violated, a Neimark-Sacker bifurcation occurs.

Comparing the stability conditions (16),(17) (19) and (22), we have the following corollary 


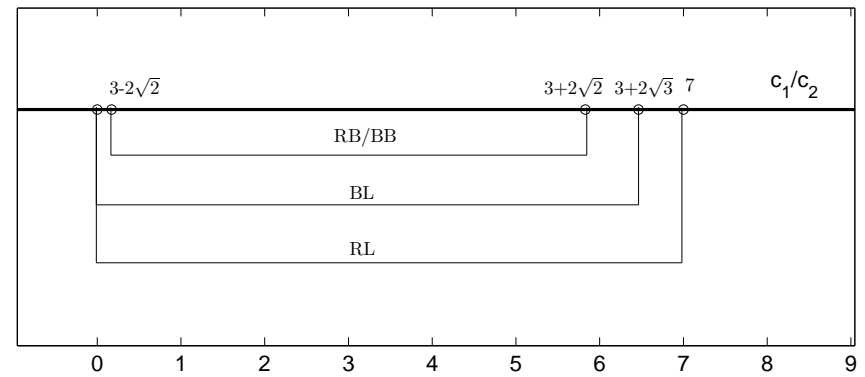

Fig. 3 Comparison of the stability intervals for models (14), (13) and (15)

Corollary 1 For any given value of one of the marginal costs $c_{i}$, we can choose the other cost $c_{-i}$ so that

- $B L, R L, B B$ and $R B$ are all stable or unstable;

- $B B$ and $R B$ are unstable while $B L$ and $R L$ are both stable;

- $B B, R B$ and $B L$ are both unstable while $R L$ is stable.

In Figure 3 we report the stability intervals with respect to $c_{1} / c_{2}$. As we can see, we have that stability can not be directly connected to the rationality degree. In particular, we have that even if in $\mathrm{RB}$ one of the players have an elevated degree of rationality, $\mathrm{RB}$ and $\mathrm{BB}$ have exactly the same stability interval. This leads to some counterintuitive situations. For instance, let us consider a duopoly consisting of two naïve firms and let us suppose that one of the firms improves its informational capabilities and acquires perfect foresight, becoming a rational firm. Then, the dynamic of the new RB duopoly would not be more stable than the previous one (BB), resulting exactly as stable (or unstable) as before. Furthermore, it can be natural to assume that if a firm becomes more rational, this can also have a positive effect on its technology, resulting in reduced marginal costs. In this case, we could have an even more counterintuitive scenario, in which the equilibrium is stable for the original $\mathrm{BB}$ duopoly but it is no more stable for the new RB duopoly.

Conversely, comparing $\mathrm{BL}$ and $\mathrm{RL}$, we have that the presence of the rational player improves the stability, as trajectories can converge for a wider range of marginal cost ratios. In particular, if the most rational player (both rational and naïve) is the most efficient, then equilibrium is stable independently of the marginal cost of the reduced rationality firm.

Remark 1 The previous stability conditions confirm and generalizes to a heterogeneous framework the results about the equilibrium stability of homogeneous oligopolies consisting of either LMA or naïve players. In [9,22], the authors show that, for an economy characterized by isoelastic demand function, the LMA mechanism gives rise to more stable dynamics with respect to the (naïve) best response one. In fact, it is shown in $[9,22]$ that considering an oligopoly of identical firms, all adopting LMA, equilibrium becomes unstable when the oligopoly consists of a least 5 firms, while for the classical best response mechanism instability already arises for 4 firms. Similarly, duopolies of LMA firms are stable for a larger interval of costs ratios than those consisting of best response firms. The stability intervals reported in Figure 3 confirm that a similar behavior arises in the present heterogeneous 
duopolies, suggesting to extend the investigation also to the case of generic size heterogeneous oligopolies of firms adopting either LMA or best response adjustment mechanisms. Moreover, all the previous results and considerations suggest that stability is connected not only to the rationality level of the firms, but it can be influenced by the way and the reactivity the least rational firm uses to adapt its strategy. To compute its best response, a LMA firm uses a conjectured linear function of a convex price function, resulting in a global underestimation of the prices, which automatically induces a more cautious adjustment.

Remark 2 To the best of our knowledge, BL model is the first example of a duopolistic competition in which equilibrium loses its stability through a NeimarkSacker bifurcation but both the firms adopt behavioral rules based on best response mechanisms, although in a reduced rationality framework. In existing literature, the Neimark-Sacker bifurcation is always related to the presence of a gradient rule, as for example shown by Angelini et al. in [4] and Tramontana in [28]. Since the difference between the destabilization through a Neimark-Sacker or a flip bifurcation is substantial, as it results in either macro-predictable (Neimark-Sacker) or possibly unpredictable chaotic (flip) dynamics, it is very important to understand whether the kind of stability loss is connected to the degree of rationality and the heuristics the firms adopt.

\subsection{Simulations}

We investigate through simulations the different qualitative behaviors of $\mathrm{RB}, \mathrm{BB}$, RL and BL models. The goal is to confirm the analysis performed in the previous section and to investigate the different quasi-periodic and chaotic behaviors of unstable equilibria.

In Figure 4 we compare the bifurcation diagrams of RB, RL and BL models (the bifurcation diagram for $\mathrm{BB}$ is essentially the same of that for RB) when $c_{2}=1$ on varying $c_{1}$. For all the simulations, the initial datum is $\left(q_{1,0}, q_{2,0}\right)=(0.01,0.2)$. We have that, when the Nash equilibrium becomes unstable, for both RB and RL models a flip bifurcation occurs, and a cascade of period doubling leads to chaos. Conversely, for BL, the dynamic is different, since when the equilibrium becomes unstable, the values of $\left(q_{1}, q_{2}\right)$ follow a quasi-periodic orbit around the equilibrium. The evolution of the closed curve attractor for $c_{1}=6.5,6.8,7,7.2$ is shown in Figure 5. It is worth noticing that in all cases both the firms adopt a best response mechanism within the same economic framework (isoelastic demand and constant marginal costs), only with different degrees of rationality. In BL model, the destabilization occurs through a quasi-periodic motion with an at least "macroscopic" level of predictability of the evolution while, on the contrary, for the other models the resulting dynamic can be completely chaotic.

\section{Convergence speed}

In Section 4 we showed how the degree of rationality affects the convergence to the Nash-Cournot equilibrium with respect to the cost ratio $c_{1} / c_{2}$. Now, we want to focus on the convergence speed of the three different models, namely we want to 

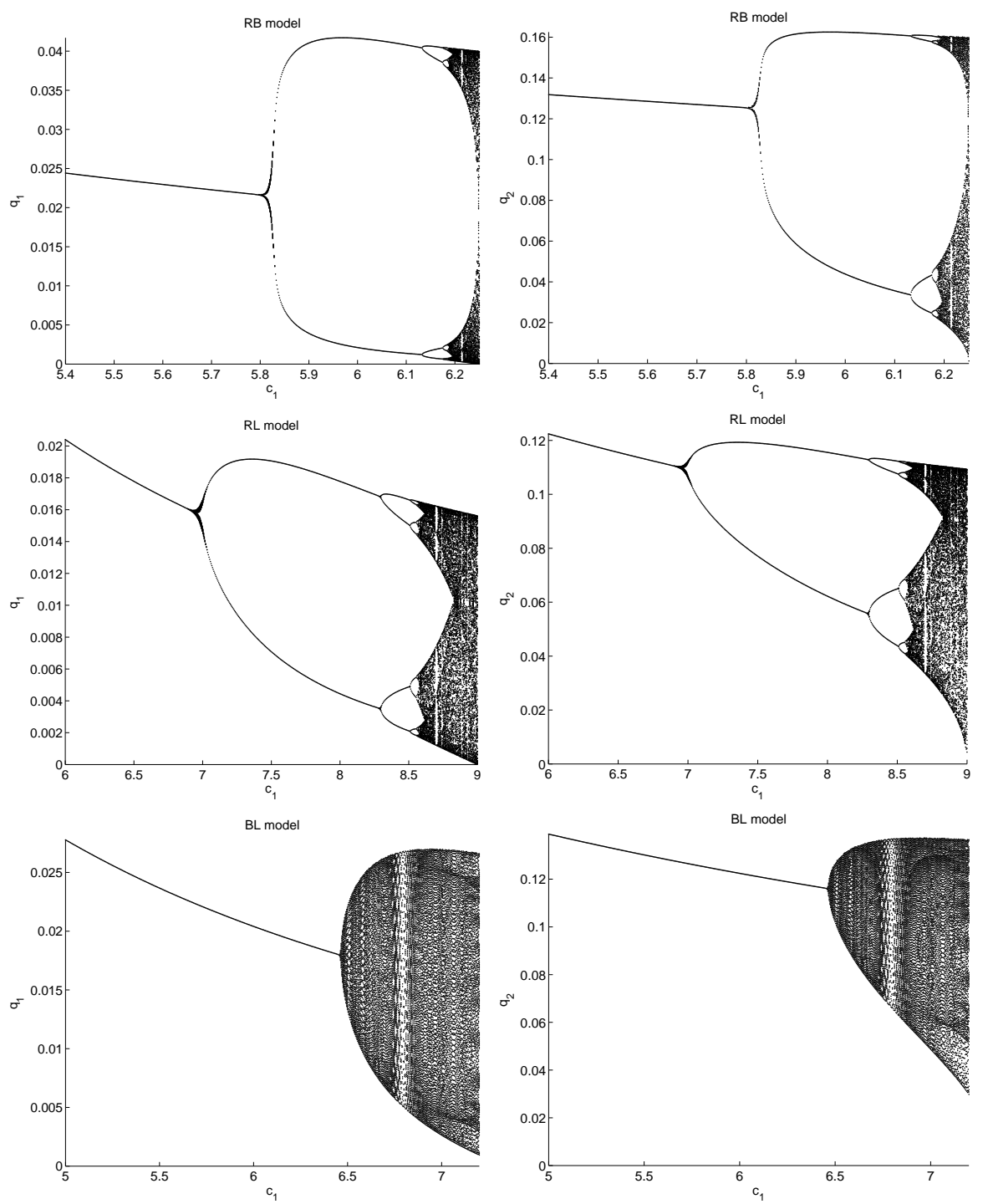

Fig. 4 Bifurcation diagrams for output levels $q_{1}$ (left column) and $q_{2}$ (right column) with $c_{2}=1$ for RB model (top), RL model (middle) and BL model (bottom). For RB and RL models stability is lost through a flip bifurcation, while BL model presents a Neimark-Sacker bifurcation.

compare the number of iterations requires to approach the equilibrium. In particular, as in the previous section we showed that the presence of a rational player is not always sufficient to improve the stability of the equilibrium, we want to investigate how convergence speed is affected by the presence of the rational player. It is evident that, since equilibrium stability depends on the largest eigenvalue, when the costs ratio approaches the instability threshold, the convergence becomes more and more slow. This means that comparing the convergence speed for models with 

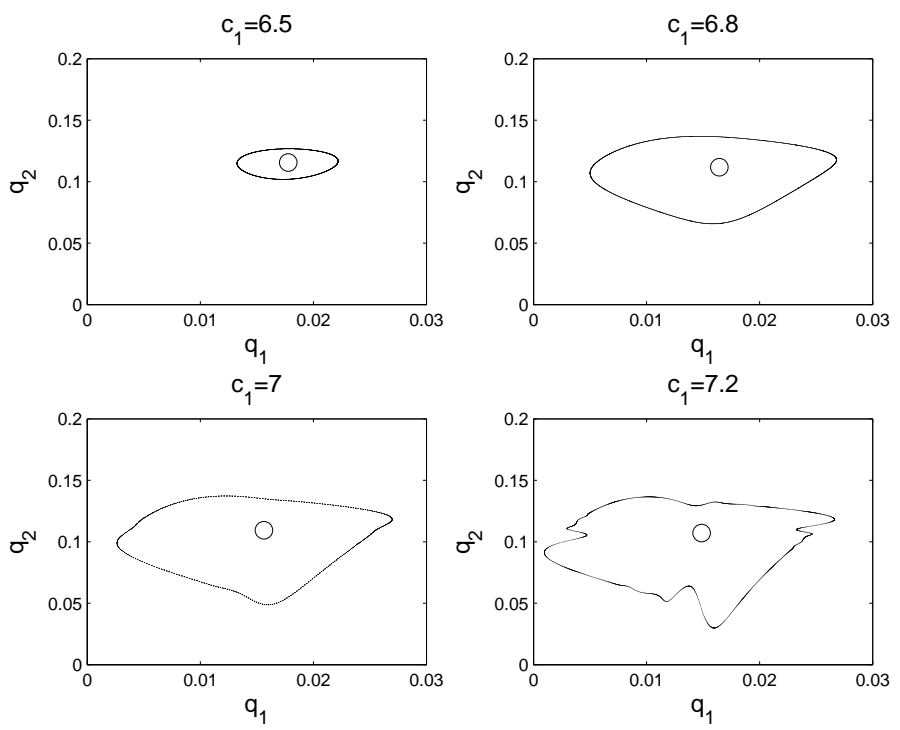

Fig. 5 Evolution of the closed curve attractor for BL model, for $c_{2}=1$ and different choices of $c_{1}$.

different stability intervals is interesting when the costs ratio is sufficiently far from both the stability thresholds of the two models. Finally, since convergence to equilibrium actually requires an infinite number of iterations, convergence speed serves as an indicator of the time required to approach the equilibrium up to a desired accuracy.

To this end, in accordance to the literature about fixed point iterations [25], for a one-dimensional map $x_{t+1}=f\left(x_{t}\right)$ we can define the asymptotic convergence factor

$$
C=\left|f^{\prime}\left(x^{N}\right)\right|
$$

where $x_{f}$ is the (stable) fixed point of the iteration map. For multidimensional maps $\mathbf{x}_{t+1}=g\left(\mathbf{x}_{t}\right)$, the previous definition becomes

$$
C=\rho\left(J\left(\mathbf{x}^{N}\right)\right)
$$

where $\rho(J)=\max _{i}\left\{\left|\lambda_{i}\right|\right\}$ is the spectral radius of the Jacobian matrix $J$ of map $g$ and $\lambda_{i}$ are its eigenvalues. In both cases, the convergence to the equilibrium is as fast as $C$ is small. When the iteration scheme converges, we have $C<1$ and, when $C \neq 0$, convergence is linear, with $C$ representing the (asymptotic) absolute error reduction, namely

$$
\left|e_{t+1}\right|<C\left|e_{t}\right|
$$

where $e_{t}=x_{t}-x^{N}$. Conversely, for $C=0$ we have a super-linear convergence.

Referring to models (12), (13), (14) and (15), setting $r=c_{1} / c_{2}$ we have the following 
Proposition 5 Provided that the respective stability conditions are satisfied, the output levels generated by $R B, B B, R L$ and $B L$ models converge to the equilibrium (3) with asymptotic convergence factors

$$
\begin{aligned}
& C_{R B}(r)=\frac{(r-1)^{2}}{4 r}, \quad C_{B B}(r)=\left|\frac{r-1}{2 \sqrt{r}}\right|, \\
& C_{R L}(r)=\left|\frac{3-r}{4}\right|, \quad C_{B L}(r)=\left|\frac{1+z}{2(r+1)}\right|,
\end{aligned}
$$

where $z$ is a positive real or complex number such that $z^{2}=r\left(-r^{2}+2 r+1\right)$.

Proof The result is a straightforward consequence of definitions (24) and (25). Factor $C_{R B}$ is simply the absolute value of (18). Factor $C_{B B}$ can be computed by noticing that

$$
J_{B B}\left(q^{N}\right)=\left(\begin{array}{cc}
0 & \frac{c_{2}-c_{1}}{2 c_{1}} \\
\frac{c_{1}-c_{2}}{2 c_{2}} & 0
\end{array}\right),
$$

whose eigenvalues are $\lambda_{1,2}= \pm i\left(c_{1}-c_{2}\right) /\left(2 \sqrt{c_{1} c_{2}}\right)$. Factor $C_{R L}$ is simply the absolute value of (20). Factor $C_{B L}$ can be obtained by computing the roots of the characteristic polynomial of (23)

$$
\lambda^{2}-\frac{c_{2}}{c_{1}+c_{2}} \lambda-\frac{c_{2}}{c_{1}+c_{2}}-\frac{c_{1}-3 c_{2}}{4 c_{2}} .
$$

Such solutions are real provided that $r\left(-r^{2}+2 r+1\right) \geq 0$, i.e. for $0 \leq r \leq 1+\sqrt{2}$, and are a complex conjugate pair for $r>1+\sqrt{2}$.

The behavior of the coefficients $(26)$ is reported in Figure 6 , for $c_{1} / c_{2} \in(3-2 \sqrt{2}, 3+$ $2 \sqrt{2}$ ), since in this range the equilibrium point is stable for all the models. We can see that BL duopoly, in which the firms use the lowest degree of rationality, is in general the slowest one. Once more, We want to draw attention on the comparison between $\mathrm{BB}$ and $\mathrm{RB}$ duopolies, as we can see that even if the stability region is exactly the same, the asymptotic convergence factor of RB is uniformly smaller than that of $\mathrm{BB}$, since we have $C_{R B}=C_{B B}^{2}$, and this results in a faster convergence speed for all costs ratios. With respect to the convergence speed, the presence of the rational firm allows for a fast approach to the equilibrium, which is true taking into account $B L$ and $R L$ too. Since the previous convergence results are valid asymptotically, we test their robustness for initial time steps through simulations. In Figure 7 we report the evolution of the relative error

$$
E_{r, t}=\frac{\left|q_{1, t}-q_{1}^{N}\right|}{q_{1}^{N}}
$$

for three particular situations. We consider $c_{2}=1$ and $c_{1}=0.9$, for which the fastest convergence is achieved for RB model, $c_{1}=2.3$, for which all the duopolies approach equilibrium with approximatively the same speed, and $c_{1}=5$, for which the fastest convergence is achieved for RL model. Such conclusions are in good agreement with the analytical results and with the plot reported in Figure 6. In fact, when the costs ratio $c_{1} / c_{2}$ is close to 1 , we have that the dynamical adjustment generated by RB model very quickly converges to the equilibrium quantity, 


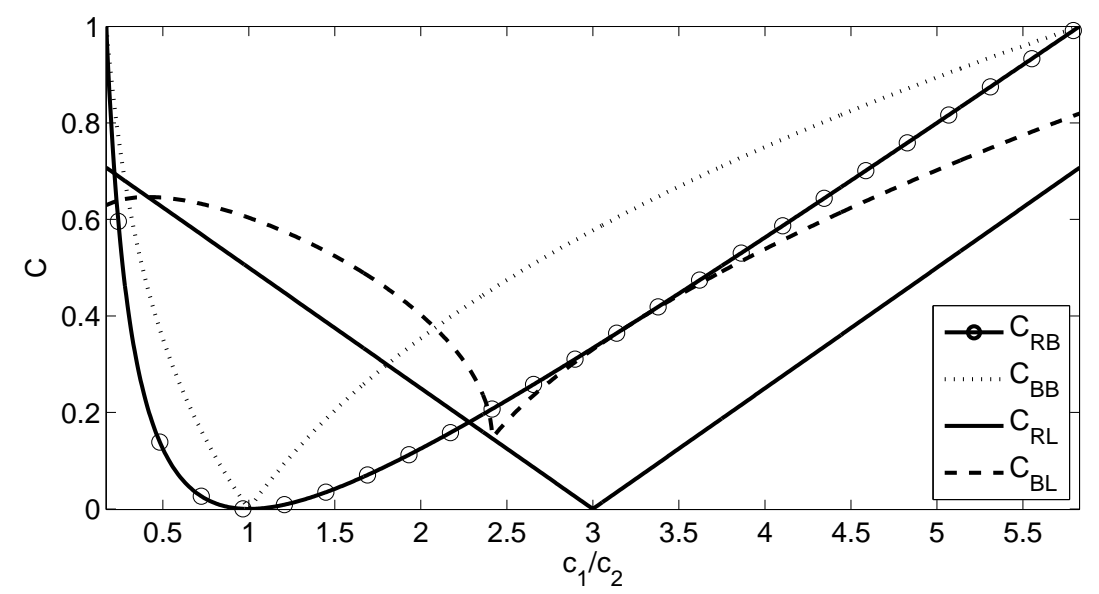

Fig. 6 Comparison of the asymptotic convergence factors. A smaller value means a faster convergence. When $c_{1} \leq c_{2}$, the output level adjustment obtained with RB model is the fastest one, while for sufficiently large $c_{1} / c_{2} \mathrm{RL}$ model has the fastest convergence.

requiring only few iterations to provide a very accurate approximation of $q_{1}^{N}$. If the rational firm is replaced by a best response one, we have that convergence, even if it is faster than those achieved by RL and BL models, is sensibly slower than that of RB. We remark that $\mathrm{RB}$ and $\mathrm{BB}$ have the same stability interval and they both provide superlinear convergence for $c_{1} / c_{2}=1\left(\right.$ as $\left.C_{R B}(1)=C_{B B}(1)=0\right)$, but they likewise have a quite different behavior for marginal costs ratios close to 1 . Conversely, both RL and BL models approach equilibrium quite slowly, behaving very similarly (top left plot of Figure 7). In the top right plot of Figure 7, we can see that when $c_{1} / c_{2}$ becomes increasingly larger than 1 , both $\mathrm{RB}$ and $\mathrm{BB}$ models converge more and more slowly, even if the presence of the rational firm in $\mathrm{RB}$ still provides the fastest convergence rate for $c_{1} / c_{2}<2.3$. Finally, as $c_{1} / c_{2}$ approaches the stability threshold of RB and BB models, RL model provides the fastest convergence. We notice that even if the stability region of $\mathrm{BL}$ is larger than that of $\mathrm{RB}$ and $\mathrm{BB}$, the convergence of $\mathrm{BL}$ toward the equilibrium is very irregular (bottom plot of Figure 7).

\section{Conclusions}

In this work we analyzed and compared several duopolies based on best response decisional mechanisms, with different degrees of rationality, in an economic setting characterized by isoelastic demand function. The analytical and simulative investigations show that the degree of rationality can have an ambiguous effect on the stability of the equilibrium, in an heterogeneous context. Such results definitely deserves further investigations. For instance, it would be interesting to generalize the previous analysis to oligopolies of generic sizes, studying the effect of composition and size on the equilibrium stability, in the case of both balanced and unbalanced marginal costs. We also want to introduce evolutionary mechanisms by means of which players can choose and change their heuristics, studying the 

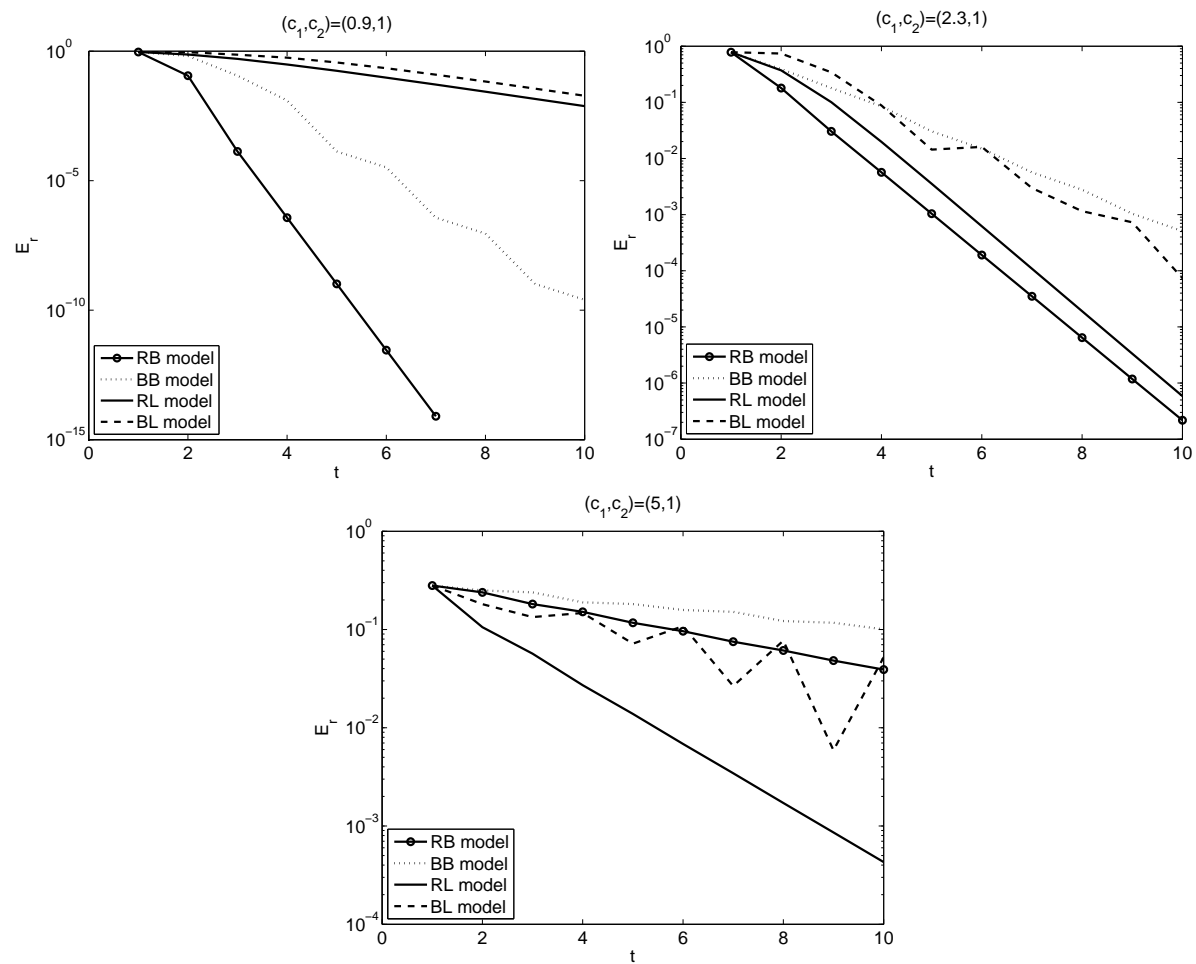

Fig. 7 Comparison of the relative errors for initial time steps and for three different coefficients choices. The behavior is in good agreement with the asymptotic results.

competition between different decisional mechanisms. Another aspect we aim to investigate concern the study in general economic settings of the more stable behavior of the LMA mechanism with respect to the naïve best response adjustment. The goal is to understand if and how such improved stability is determined by the particular form of the price function.

In our contribution we also provided an example of duopoly model in which equilibrium loses its stability through a Neimark-Sacker bifurcation and in which the decisional mechanisms are not based on a gradient-like behavioral rule, but instead on a best response method. In future works, we aim to investigate and compare different models to understand the reasons that cause the destabilization through either Neimark-Sacker or flip bifurcation, in particular with respect to the kind of behavioral rule, as this aspect has economic relevance for the predictability of the trajectories of the output levels in unstable regimes.

\section{References}

1. Agiza, H.N., Elsadany, A.A.: Nonlinear dynamics in the Cournot duopoly game with heterogeneous players. Physica A 320, 512-524 (2003)

2. Agiza, H.N., Elsadany, A.A.: Chaotic dynamics in nonlinear duopoly game with heterogeneous players. Appl. Math. Comput. 149(3), 843-860 (2004) 
3. Agiza, H.N., Hegazi, A.S., Elsadany, A.A.: Complex dynamics and synchronization of a duopoly game with bounded rationality. Math. Comput. Simul. 58(2), 133-146 (2002)

4. Angelini, N., Dieci, R., Nardini, F.: Bifurcation analysis of a dynamic duopoly model with heterogeneous costs and behavioural rules. Math. Comput. Simul. 79(10), 3179-3196 (2009)

5. Askar, S.S.: The rise of complex phenomena in Cournot duopoly games due to demand functions without inflection points. Commun. Nonlinear Sci. Numer. Simul. 19(6), 19181925 (2014)

6. Bischi, G.I., Gallegati, M., Naimzada, A.: Symmetry-breaking bifurcations and representative firm in dynamic duopoly games. Ann. Oper. Res. 89, 253-272 (1999)

7. Bischi, G.I., Kopel, M., Naimzada, A.: On a rent-seeking game described by a noninvertible iterated map with denominator. Nonlinear Anal. 47(8), 5309 - 5324 (2001)

8. Bischi, G.I., Naimzada, A.: Global analysis of a dynamic duopoly game with bounded rationality. In: J. Filar, V. Gaitsgory, K. Mizukami (eds.) Adv. in Dyn. Games and Appl., pp. 361-385. Shonan Village Ctr (2000). 7th Int. Symp. on Dyn. Games and Appl.

9. Bischi, G.I., Naimzada, A., Sbragia, L.: Oligopoly games with local monopolistic approximation. J. Econ. Behav. Organ. 62(3), 371-388 (2007)

10. Cavalli, F., Naimzada, A.: A cournot duopoly game with heterogeneous players: nonlinear dynamics of the gradient rule versus local monopolistic approach. Appl. Math. Comput. 249, 382-388 (2014)

11. Cavalli, F., Naimzada, A., Tramontana, F.: Nonlinear dynamics and global analysis of an heterogeneous cournot duopoly with a local monopolistic approach versus a gradient rule with endogenous reactivity (2014). Commun. Nonlinear Sci. Numer. Simul., in press, available online doi:10.1016/j.cnsns.2014.11.013 (2014)

12. Cournot, A.A.: Researches into the principles of the theory of wealth. Engl. Trans., Irwin Paper Back Classics in Economics (1963)

13. Den-Haan, W.J.: The importance of the number of different agents in a heterogeneous asset-pricing model. J. Econ. Dyn. Control 25(5), 721-746 (2001)

14. Dubiel-Teleszynski, T.: Nonlinear dynamics in a heterogeneous duopoly game with adjusting players and diseconomies of scale. Commun. Nonlinear Sci. Numer. Simul. 16(1), 296 - 308 (2011)

15. Elsadany, A.A., Tramontana, F.: Heterogeneous triopoly game with isoelastic demand function. Nonlinear Dyn 68(1-2), 187-193 (2012)

16. Gao, X., Zhong, W., Mei, S.: Nonlinear cournot oligopoly games with isoelastic demand function: The effects of different behavior rules. Commun. Nonlinear Sci. Numer. Simul. $\mathbf{1 7}(12), 5249-5255(2012)$

17. Leonard, D., Nishimura, K.: Nonlinear dynamics in the cournot model without full information. Ann. Oper. Res. 89, 165-173 (1999)

18. Li, T., Ma, J.: The complex dynamics of rçompetition models of three oligarchs with heterogeneous players. Nonlinear Dyn. 74(1-2), 45-54 (2013)

19. Ma, J., Wu, F.: The application and complexity analysis about a high-dimension discrete dynamical system based on heterogeneous triopoly game with multi-product. Nonlinear Dyn. 77(3), 781-792 (2014)

20. Naimzada, A., Ricchiuti, G.: Monopoly with local knowledge of demand function. Econ. Model. 28(1-2), 299-307 (2011)

21. Naimzada, A., Sbragia, L.: Oligopoly games with nonlinear demand and cost functions: two boundedly rational adjustment processes. Chaos, Solitons Fractals 29(3), $707-722$ (2006)

22. Naimzada, A., Tramontana, F.: Controlling chaos through local knowledge. Chaos, Solitons Fractals 42(4), $2439-2449$ (2009)

23. Poston, T., Stewart, I.: Catastrophe theory and its applications. London: Pitman Ltd. (1978)

24. Puu, T.: Chaos in duopoly pricing. Chaos, Solitons Fractals 1(6), 573 - 581 (1991)

25. Quarteroni, A., Sacco, R., Saleri, F.: Numerical mathematics, Texts in Applied Mathematics, vol. 37, second edn. Springer-Verlag, Berlin (2007)

26. Rand, D.: Exotic phenomena in games and duopoly models. J. Math. Econom. 5(2), 173 $-184(1978)$

27. Silvestre, J.: A model of general equilibrium with monopolistic behavior. J. Econ. Theory 16, 425-442 (1977)

28. Tramontana, F.: Heterogeneous duopoly with isoelastic demand function. Econ. Model. 27(1), 350-357 (2010)

29. Tuinstra, J.: A price adjustment process in a model of monopolistic competition. Int. Game Theory Rev. 6(3), 417-442 (2004) 